\title{
Perioperative temporary mechanical circulatory support with Impella in cardiac surgery patients
}

\author{
Mateo Marin-Cuartas ${ }^{1}$, Katharina Wehrmann ${ }^{1}$, Michael Höbartner ${ }^{1}$, Sven Lehmann ${ }^{1}$, \\ Christian Etz ${ }^{1}$, Diyar Saeed ${ }^{1}$, and Michael Borger ${ }^{1}$ \\ ${ }^{1}$ Herzzentrum Leipzig Universitätsklinik
}

June 2, 2021

\begin{abstract}
Background: The benefits of perioperative mechanical circulatory support (MCS) in cardiac surgery patients are still uncertain. This study aims to review early outcomes of perioperative temporary MCS using the Impella device in cardiac surgery patients. Methods: Retrospective, single center analysis in cardiac surgery patients presenting with cardiogenic shock (CS) in whom Impella was used for perioperative temporary MCS, whether as single device therapy or as left ventricular (LV) venting strategy for concomitant extra corporeal membrane oxygenation (ECPELLA). Study outcomes were 30-day mortality and occurrence of complication composite outcome. Results: Between 2016 and 2019, a total of 33 consecutive patients were supported with Impella [single-device therapy in $19(57.6 \%)$ patients and ECPELLA in 14 (42.4\%) patients]. The 30-day mortality of Impellaalone and ECPELLA groups was $15.8 \%$ and $50.0 \%(\mathrm{P}=0.03)$. The 30-day mortality according to pre-, intra- and postoperative implantation was $12.5 \%, 60.0 \%$ and $28.6 \%(\mathrm{P}=0.04)$, and it was significantly lower in those patients in whom a left ventricular assist device was implanted in comparison to all other surgical procedures $(\mathrm{P}<0.01)$. The complication composite outcome occurred more frequently after axillary implantation in comparison to femoral Impella $(\mathrm{P}=0.05)$ due to higher stroke rates $(\mathrm{P}=0.03)$. Bleeding requiring surgical re-exploration was more frequent in the ECPELLA than in the Impella-alone group [1 $(3.0 \%)$ vs $5(15.1 \%) ; \mathrm{P}=0.03]$. Conclusions: Temporary MCS with Impella is associated with high complication and mortality rates. However, preoperative use of Impella as single-device temporary MCS is associated with lower mortality rates and is a reasonable alternative as bridge-to-decision strategy for acutely decompensated patients.
\end{abstract}

Perioperative temporary mechanical circulatory support with Impella in cardiac surgery patients

Mateo Marin-Cuartas, MD*; Katharina Wehrmann, MD*; Michael Höbartner, MD; Sven Lehmann, MD, PhD, MBA; Christian D. Etz, MD, PhD; Diyar Saeed, MD, PhD; Michael A. Borger, MD, PhD.

* Both authors contributed equally to this work

University Department of Cardiac Surgery, Leipzig Heart Center, Leipzig, Germany

Corresponding Author:

Dr. Mateo Marin-Cuartas

University Department of Cardiac Surgery

Heart Center Leipzig

Struempellstrasse 39

04289 Leipzig, Germany

Phone: +49-341-865-0 
Fax: +49-341-865-1452

Email: mateomarinc@hotmail.com

Word count: 4494

\title{
Disclosures
}

MMC discloses receiving speakers' honoraria from CytoSorbents GmbH. MAB discloses that his hospital receives speakers' honoraria and/or consulting fees on his behalf from Edwards Lifesciences, Medtronic, Abbott, and CryoLife. The remaining authors have no conflicts of interest or financial relationships with industry to disclose.

Funding: None

\begin{abstract}
Background: The benefits of perioperative mechanical circulatory support (MCS) in cardiac surgery patients are still uncertain. This study aims to review early outcomes of perioperative temporary MCS using the Impella device in cardiac surgery patients.
\end{abstract}

Methods: Retrospective, single center analysis in cardiac surgery patients presenting with cardiogenic shock (CS) in whom Impella was used for perioperative temporary MCS, whether as single device therapy or as left ventricular (LV) venting strategy for concomitant extra corporeal membrane oxygenation (ECPELLA). Study outcomes were 30-day mortality and occurrence of complication composite outcome.

Results: Between 2016 and 2019, a total of 33 consecutive patients were supported with Impella [singledevice therapy in $19(57.6 \%)$ patients and ECPELLA in $14(42.4 \%)$ patients]. The 30-day mortality of Impella-alone and ECPELLA groups was $15.8 \%$ and $50.0 \%(\mathrm{P}=0.03)$. The 30-day mortality according to pre-, intra- and postoperative implantation was $12.5 \%, 60.0 \%$ and $28.6 \%(\mathrm{P}=0.04)$, and it was significantly lower in those patients in whom a left ventricular assist device was implanted in comparison to all other surgical procedures $(\mathrm{P}<0.01)$. The complication composite outcome occurred more frequently after axillary implantation in comparison to femoral Impella $(\mathrm{P}=0.05)$ due to higher stroke rates $(\mathrm{P}=0.03)$. Bleeding requiring surgical re-exploration was more frequent in the ECPELLA than in the Impella-alone group [1 $(3.0 \%)$ vs $5(15.1 \%) ; \mathrm{P}=0.03]$.

Conclusions: Temporary MCS with Impella is associated with high complication and mortality rates. However, preoperative use of Impella as single-device temporary MCS is associated with lower mortality rates and is a reasonable alternative as bridge-to-decision strategy for acutely decompensated patients.

Key words: Mechanical Circulatory Support; Cardiogenic Shock; Impella; LVAD; Heart Transplantation

\section{Abbreviations}

CS - cardiogenic shock

ECMO - extracorporeal membrane oxygenation

ECPELLA - ECMO + Impella

MCS - mechanical circulatory support

LV - left ventricular

IABP - intra-aortic balloon pump

IQR - interquartile range

\section{Introduction}

Mechanical circulatory support (MCS) has evolved markedly over the last decades. Temporary MCS strategies such as extracorporeal membrane oxygenation (ECMO) and Impella (Abiomed, Danvers, MA) are 
nowadays widely employed for the treatment of patients with cardiogenic shock (CS). Both temporary MCS options allow immediate resuscitation and hemodynamic stabilization with a potential end-organ recovery. The availability and accessibility of these systems have significantly increased during the recent years leading to a rapid rise in the number of implanted devices around the world. Nonetheless, the exponential increase in the use of these systems is not necessarily associated with improved survival, since there are many factors determining the outcome in patients with CS. The success of MCS strategies not only depends on the availability of the devices, but also diverse expertise in the management of this complex patient population including multidisciplinary treatment of severe complications, which often require huge amounts of resources.

Among many of the critical aspects to consider while managing patients with CS under temporary MCS is the so-called "venting" of the left ventricle. ECMO therapy provides adequate amount of flow and simultaneous pulmonary support. However, ECMO flow is not physiologic, and it increases the left ventricular (LV) afterload. Venting is therefore frequently necessary for adequate LV volume unloading, thereby preventing increased LV end-diastolic pressure and subsequent myocardial ischemia and pulmonary edema in patients under ECMO support. LV venting has been demonstrated to be associated with improved survival but is not used in all ECMO patients.

Several LV venting strategies have been described: use of inotropic agents to facilitate ejection through the aortic valve, use of intra-aortic balloon pump (IABP), implantation of a surgical vent, atrial septostomy, and percutaneous implantation of a catheter vent through the jugular vein into the pulmonary artery. Some authors have proposed the combined use of Impella and ECMO (also known as ECPELLA) to improve LV venting. The ECPELLA strategy is associated with higher ECMO weaning rates and successful bridging to permanent ventricular assist device or cardiac transplant. However, it is also associated with a high rate of major complications such as hemolysis and limb ischemia.

Clinical trials on Impella as temporary MCS are based on a very heterogeneous populations with different types of CS, and its use in cardiac surgery patients whether a single device therapy or as LV venting strategy is supported by limited evidence. Moreover, most of the clinical trials are based on comparisons of ECMO versus ECPELLA, but there is a lack of studies comparing ECPELLA versus Impella alone. Therefore, the aim of this study is to present a series of cardiac surgery patients presenting with perioperative CS and to review their early outcomes after temporary MCS with Impella, including a comparative subgroup analysis of isolated Impella versus ECPELLA. Given the small sample size of the current cohort, this study is limited to a descriptive report. Statistical comparisons are purely exploratory.

\section{Patients and Methods}

\section{Ethical statement}

This study was approved by the ethics committee of the Faculty of Medicine at the University of Leipzig and individual patient informed consent was waived.

\section{Study cohort}

Between October 2016 and June 2019, a total of 33 consecutive adult cardiac surgery patients from our institution presenting with CS in whom Impella was used for perioperative temporary MCS, whether as isolated therapy or as LV-venting strategy for concomitant ECMO were included in the present study. The preoperative patient characteristics, intra-operative data, and postoperative outcomes were prospectively collected and entered into a computerized database and were retrospectively analyzed.

\section{Mechanical circulatory support strategies}

All patients included in the study were supported with Impella whether as single-device therapy or as LVventing for concomitant ECMO (ECPELLA). Patients with Impella 2.5, Impella 3.5 and Impella 5.0 devices as well as patients with central and peripheral ECMO were included in the analysis. Pre- and postoperative implantation of any of both MCS strategies was performed in patients with perioperative refractory CS. Intraoperative implantation of any of both MCS strategies was performed if weaning off from CPB was 
not possible due to hemodynamic instability. The selection of the support strategy and the type of Impella device implanted was determined by the timing and clinical presentation of CS, as well as according to specific patient characteristics such peripheral vascular disease and timing of intervention (i.e., emergent or urgent; pre-, intra- or postoperative). Finally, the decision was made by the whole surgical time in a specific case-to-case basis. A detailed description of the implantation technique of both, Impella and ECMO, is provided in the Supplementary Appendix .

In ECPELLA pantients, the implantation of Impella and ECMO occurred simultaneously during the same procedure. For both, Impella alone and ECPELLA, once bleeding was ruled out, anticoagulation was achieved with continuous intravenous infusion of heparin with a goal partial thromboplastin time of 40-60 seconds. The ideal flow rate in patients with ECPELLA was based on the summatory of the total ECMO and Impella flow and it was calculated according to the body surface area. Moreover, for both Impella alone and ECPELLA, the Impella device was always running at the highest possible flow according to the Impella type until weaning was started.

\section{Study outcomes and definition of explanatory variables}

The primary outcome of the study was 30-day mortality. The secondary study outcome was a complication composite outcome during the initial 30 postoperative days and was defined as occurrence of at least one of the following: major bleeding requiring surgical re-exploration of the thorax, active bleeding at the Impella insertion site, limb ischemia, Impella thrombosis, stroke, arteriovenous fistula, arterial pseudoaneurysm, and groin/axillary hematoma.

Definitions of perioperative CS and additional postoperative outcomes such as acute kidney injury and acute hepatic dysfunction are provided in the Supplementary Appendix .

\section{Statistical analysis}

Categorical variables are expressed as frequencies and percentages throughout the manuscript. Continuous variables are expressed as mean \pm standard deviation for normally distributed variables, and as median and interquartile range (IQR) for non-normally distributed variables. Student's T-test and/or Fisher's exact test were used for pair-wise comparison of variables. One-way analysis of variance (ANOVA) was used to assess for differences between 3 or more variables. Statistical significance was defined at $P<0.05$ for all tests. A posthoc comparison of the 30-day mortality according to the type of surgery is provided in the Supplementary Appendix . Perioperative variables that had a univariable value of $P<0.25$ or those judged to be clinically important were submitted to a multivariable logistic regression model by backward stepwise selection to determine the independent predictors of 30-day mortality, which are expressed as odds ratios (OR) and $95 \%$ confidence intervals (95\% CI). All analyses were performed using SPSS Statistics (IBM Corp. Released 2017. IBM SPSS Statistics for Macintosh, Version 25.0. Armonk, NY: IBM Corp.).

\section{Results}

\section{Baseline demographics}

Between October 2016 and June 2019, a total of 33 consecutive cardiac surgery patients presenting with perioperative CS underwent temporary MCS with the Impella device. Amongst them, a total of 19 (57.6\%) patients were supported with Impella as single-device therapy (Impella-alone group) and 14 (42.4\%) patients were supported with Impella as LV venting during concomitant ECMO therapy (ECPELLA group). Preoperative patient characteristics are depicted in Table 1.No statistically significant difference was observed after comparison of the patients' baseline characteristics according to the different study subgroups Table 2.

\section{Implantation details and Impella-related information}

Among the Impella-alone group, a total of $9(27.3 \%)$ patients and $10(30.3 \%)$ were supported with Impella 3.5 and Impella 5.0, respectively. Patients from the ECPELLA group were vented with either Impella 2.5 [10 $(30.3 \%)$ patients] or Impella 3.5 [4 (12.1\%) patients]. Further implantation details are presented in Table 3. 


\section{Postoperative outcomes}

A summary of the early postoperative outcomes is depicted inTable 4. The overall 30-day mortality in the entire study cohort was $30.3 \%$. Thirty-day mortality of the Impella-alone group was significantly lower than the ECPELLA group (15.8\% versus $50 \%, \mathrm{P}=0.03)$, (Figure 1 ). The 30-day mortality according to timing of MCS-device implantation was $12.5 \%, 60.0 \%$ and $28.6 \%$ in patients who underwent pre-, intra- and postoperative implantation $(\mathrm{P}=0.04)$, respectively (Figure 2 ). Multivariable logistic regression analysis identified age $>65$ years (OR:6.2; 95\% CI $1.1-36.2 ; P=0.04$ ) as the only independent predictor for 30-day mortality. Other relevant clinical complications such as bleeding and stroke were not identified as predictors of 30-day mortality.

Thirty-day mortality rates according to the type of surgery are depicted in Figure 3 . A total of 12 (36.4\%) patients who underwent LVAD implantation required perioperative temporary MCS and presented a 30day mortality rate of $0 \%(\mathrm{P}<0.01$ in comparison to the mortality of the remaining patients from the whole cohort; post-hoc analysis is provided in the Supplementary Appendix ). Among them, 10 (30.3\%) patients were supported with Impella alone and $2(6.1 \%)$ with ECPELLA. Moreover, among LVAD patients, the temporary MCS was implanted preoperatively in $10(30.3 \%)$ patients, intraoperatively in $1(3.0 \%)$ patient, and postoperatively in $1(3.0 \%)$ patient.

The complication composite outcome occurred in 15 (45.4\%) patients from the entire study cohort. Figures 4 A - C summarize the complication composite outcome according to the temporary MCS strategy, timing of implantation, and type of surgery. Major bleeding requiring surgical re-exploration of the thorax was more frequently observed among patients with ECPELLA in comparison to patients with Impella alone [1 $(3.0 \%)$ vs $5(15.1 \%) ; \mathrm{P}=0.03]$. A trend towards a more frequent occurrence of the composite outcome was observed among patients with axillary Impella in comparison to patients with femoral Impella [Axillary: $6(18.2 \%)$ patients; Femoral: $9(27.3 \%)$ patients; $(\mathrm{P}=0.05)]$. This is explained through higher stroke rates among axillary Impella patients; a total of $4(12.1 \%)$ patients presented postoperative stroke, among them $3(9.1 \%)$ patients were supported with axillary Impella and $1(3.0 \%)$ patient was supported with femoral Impella $(\mathrm{P}=0.03)$. Among those patients with postoperative ischemic stroke, only $1(3.0 \%)$ patient presented with Impella thrombosis. A summary of the study outcomes and procedural aspects is depicted in

\section{Table 5.}

\section{Discussion}

To the best of our knowledge, this is the first reported series of only cardiac surgery patients with perioperative CS in whom Impella was used as temporary MCS. Given the small cohort size, this study is limited to a descriptive report. Statistical comparisons are purely exploratory. The main findings are:

1. Impella as LV-venting strategy in patients under ECMO support is associated with high complication and mortality rates.

2. Intraoperative implantation of Impella, both as LV venting strategy or as single-device temporary MCS, is associated with poor outcomes.

3. Preoperative temporary MCS with Impella as single-device therapy is a reasonable alternative as bridge-to-decision strategy (e.g., bridge-to-LVAD or bridge-to-transplantation) for patients with decreased LV function presenting with CS.

In patients with CS requiring MCS, LV-venting is associated with improved survival. Nonetheless, the different strategies for $\mathrm{LV}$-venting have considerable limitations. There is no convincing evidence supporting the concomitant use of IABP in ECMO patients. The use of surgical vents in patients under ECMO support has been advocated by some authors, but this strategy is associated with higher bleeding and complication rates as well as frequent thrombosis of the venting cannula. Atrial septostostomy is a promising alternative for LV-venting, but it requires the iatrogenic creation of a hemodynamically relevant shunt between both atrial chambers. Some clinicians advocate central cannulation for ECMO instead of peripheral cannulation, resulting in superior LV-unloading based on the theoretical advantage of more physiologic (antegrade) blood 
flow in the proximal aorta. However, available studies have failed to demonstrate any real clinical advantage of central over peripheral ECMO.

All of the above-described drawbacks and uncertainties of various LV-venting techniques has motivated some cardiac surgery groups to start using Impella as a venting alternative. Moreover, since Impella is a percutaneous device, it makes it an attractive option to reduce the invasiveness in surgical patients with CS who require prolonged temporary MCS as bridge-to-decision or a more definitive therapy. A recent systematic review published by Vallabhajosyula et al.concluded that the use of ECPELLA was associated with higher weaning from ECMO and bridging to permanent LVAD or cardiac transplantation. These positive results may encourage cardiac surgeons to employ this strategy for surgical patients. However, this study is based on general population patients with CS and not on cardiac surgery patients, who are more prone to bleeding and other severe complications. Our current results focused only on cardiac surgery patients receiving an Impella device and showed high morbidity and mortality rates. The 30-day mortality among those patients in whom the Impella was implanted intra- and postoperatively was $47 \%$. This is not markedly different from our 30-day mortality rate of $57 \%$ observed among patients with postcardiotomy CS requiring ECMO support. Moreover, this small survival difference needs to be placed in the context that the Impella device is more expensive and technically more complex than isolated ECMO therapy and was associated with a high incidence of major complications in the current study. For example, the ECPELLA strategy in our study was associated with an increased frequency of major bleeding requiring surgical re-exploration.

One patient group that had a lower 30-day mortality in the current study was those patients who underwent preoperative Impella implantation. However, the non-fatal complication rates were similarly high amongst groups, irrespective of the timing of implantation. Interestingly, the 30-day mortality rate of those CS patients receiving a preoperative Impella as bridge-to-decision and subsequently underwent LVAD implantation was $0 \%$. Moreover, the 30-day mortality among those patients with preoperative Impella MCS who underwent heart transplantation was also $0 \%$. Therefore, we believe that the use of an Impella device should be advised as a preoperative single-device temporary MCS alternative, which results in end-organ stabilization in acutely decompensated patients. Finally, given the high complication and mortality rates, the complex handling and the economic costs associated with Impella and ECPELLA therapy, and the additional risk factor of age older than 65 years, perioperative temporary MCS with Impella is not advised in elderly patients.

In conclusion, intra- and postoperative implantation of Impella, both as LV-venting for ECMO or as singledevice temporary MCS, is associated with high complication and mortality rates. Preoperative use of Impella as single-device temporary MCS is a reasonable alternative as bridge-to-decision strategy (e.g., bridge-toLVAD) for acutely decompensated patients with decreased LV function presenting with CS.

\section{Study limitations}

This study, being a single center experience and retrospective in nature, is subject to inherent biases. The study cohort is small; thus, interpretation of various statistical analyses should be addressed with caution. Moreover, the small number of patients considerably limits the feasibility of more advanced statistical methods. Hence, this study is limited to a descriptive report. Statistical comparisons are only exploratory, and the resulting conclusions must be interpreted with caution. However, our experience with Impella and ECPELLA in this very specific subset of cardiac surgery patients is considerable in comparison to smaller centers. Being a tertiary referral center that performs large volumes of cardiac operations, our reported outcomes may not be applicable to all institutions, especially those that have limited experience in managing temporary MCS. Finally, some additional data such as detailed laboratory parameters, doses of vasoactive medications, hourly register of MCS flow rates or echocardiographic follow-up during temporary MCS were not retrospectively available in our database.

Tables

Table 1. Demographic and preoperative characteristics 
Gender

Female, n (\%)

Male, n (\%)

Age - years, median (IQR)

LVEF - \%, median (IQR)

Atrial fibrillation, n (\%)

Arterial hypertension, n (\%)

Diabetes mellitus, n (\%)

Chronic kidney disease, n (\%)

COPD, n (\%)

Peripheral artery disease, n (\%)

Hyperlipidemia, n (\%)

Dilated cardiomyopathy, n (\%)

Ischemic cardiomyopathy, n (\%)

Pacemaker, n (\%)

Previous cardiac surgery, n (\%)

Mechanical ventilation, $\mathrm{n}(\%)$

Acute kidney injury, n (\%)

Preoperative cardiogenic shock, n (\%)

CAD, n (\%)

Infective endocarditis, n (\%)

CAD coronary artery disease; COPD chronic obstructive pulmonary disease; IQR interquartile range; LVEF left ventrict

Table 2. Comparison of preoperative characteristics according to MCS strategy

Female gender, $\mathrm{n}(\%)$

Age - years, median (IQR)

LVEF - \%, median (IQR)

Atrial fibrillation, $\mathrm{n}(\%)$

Arterial hypertension, $\mathrm{n}(\%)$

Diabetes mellitus, $\mathrm{n}(\%)$

Chronic kidney disease, n (\%)

COPD, n $(\%)$

Peripheral artery disease, n (\%)

Previous cardiac surgery, n (\%)

Mechanical ventilation, $\mathrm{n}(\%)$

Acute kidney injury, n (\%)

Preoperative CS, n (\%)

CAD, n $(\%)$

Infective endocarditis, n (\%)

CAD coronary artery disease; COPD chronic obstructive pulmonary disease; CS cardiogenic shock; LVAD left ventricula

Table 3. Impella related information and implantation details

Type of MCS

Impella $2.5+$ central ECMO, n $(\%)$

Impella $2.5+$ peripheral ECMO, n (\%)
Table 3. Impella related informat $\mathrm{n}=33$

$7(21.2)$

$3(9.1)$ 
Impella $3.5+$ central ECMO, n (\%)

$3(9.1)$

Impella $3.5+$ peripheral ECMO, n $(\%)$

$1(3.0)$

Impella 3.5 single device, n (\%)

$9(27.3)$

Impella 5.0 single device, n (\%)

Implantation site

Axilary insertion, n (\%)

$8(24.2)$

Femoral insertion, n (\%)

$25(75.7)$

Timing for implantation

Preoperative implantation, n (\%)

$16(48.5)$

Intraoperative implantation, n (\%)

$10(30.3)$

Postoperative implantation, n (\%)

$7(21.2)$

Setting for implantation

Urgent, n (\%)

$11(33.3)$

Emergent, n (\%)

ECMO extracorporeal membrane oxygenation; MCS mechanical circulatory support

ECMO extracorporeal membrane

Table 4. Early postoperative outcomes

30-day mortality - \%

Complication composite outcome*, n (\%)

Ischemic stroke, n (\%)

Impella thrombosis, n (\%)

Limb ischemia, n (\%)

Arteriovenous fistula, n (\%)

Axillary hematoma, n (\%)

Groin hematoma, n (\%)

Arterial pseudoaneurysm at insertion site, n (\%)

Active bleeding at insertion site, $\mathrm{n}(\%)$

Major bleeding requiring surgical re-exploration, n (\%)

Other complications

Acute kidney injury, n (\%)

Acute hepatic dysfunction, n (\%)

Sepsis, n (\%)

ARDS, n (\%)

Intracranial bleeding, n (\%)

Gastrointestinal ischemia, n (\%)

Occurrence of at least one of the following: major bleeding requiring surgical re-exploration of the thorax, active bleeding a

Table 5. Summary of study outcomes and comparison of procedural aspects

\begin{tabular}{llc}
\hline Comparison & 30-day mortality $\%$ & $\begin{array}{c}\text { Complica } \\
\text { outcom }\end{array}$ \\
$\begin{array}{l}\text { Temporary MCS strategy } \\
\text { Impella alone (n=19) }\end{array}$ & 15.8 & $8(42.1)$ \\
ECPELLA (n=14) & 50.0 & $7(50.0)$ \\
P-Value & $\mathbf{0 . 0 3}$ & 0.65 \\
Timing of implantation & 12.5 & $7(43.7)$ \\
$\begin{array}{l}\text { Preoperative implantation } \\
\text { (n=16) }\end{array}$ & &
\end{tabular}




\begin{tabular}{|c|c|c|}
\hline $\begin{array}{l}\text { Intraoperative implantation } \\
(\mathrm{n}=10)\end{array}$ & 60.0 & $4(40.0)$ \\
\hline $\begin{array}{l}\text { Postoperative implantation } \\
(\mathrm{n}=7)\end{array}$ & 28.6 & $4(57.1)$ \\
\hline P-Value & 0.04 & 0.77 \\
\hline Impella implantation site & & \\
\hline Impella axillary insertion $(\mathrm{n}=8)$ & 12.5 & $6(75.0)$ \\
\hline $\begin{array}{l}\text { Impella femoral insertion } \\
(\mathrm{n}=25)\end{array}$ & 36.0 & $9(36.0)$ \\
\hline $\begin{array}{l}\text { P-Value } \\
\text { Type of surgery }\end{array}$ & 0.21 & 0.05 \\
\hline LVAD Implantation $(\mathrm{n}=12)$ & 0 & $7(58.3)$ \\
\hline $\operatorname{CABG}(\mathrm{n}=11)$ & 45.4 & $5(45.4)$ \\
\hline Valve surgery $(\mathrm{n}=5)$ & 60.0 & $2(40.0)$ \\
\hline Heart transplantation $(\mathrm{n}=3)$ & 0 & $1(33.3)$ \\
\hline Other operation $(\mathrm{n}=2)^{*}$ & 100 & $0(0)$ \\
\hline P-Value & $<0.01$ & 0.61 \\
\hline $\begin{array}{l}1(3.0 \%) \text { patient underwent } \\
\text { ventricle septal defect closure } \\
\text { and } 1(3.0 \%) \text { patient underwent } \\
\text { replacement of the ascending } \\
\text { aorta. CABG coronary artery } \\
\text { bypass grafting; ECMO } \\
\text { extracorporeal membrane } \\
\text { oxygenation; LVAD left } \\
\text { ventricular assist device; MCS } \\
\text { mechanical circulatory support. }\end{array}$ & $\begin{array}{l}* 1(3.0 \%) \text { patient underwent } \\
\text { ventricle septal defect closure } \\
\text { and } 1(3.0 \%) \text { patient underwent } \\
\text { replacement of the ascending } \\
\text { aorta. CABG coronary artery } \\
\text { bypass grafting; ECMO } \\
\text { extracorporeal membrane } \\
\text { oxygenation; LVAD left } \\
\text { ventricular assist device; MCS } \\
\text { mechanical circulatory support. }\end{array}$ & $\begin{array}{l}* 1(3.0 \%) \text { patient underwent } \\
\text { ventricle septal defect closure } \\
\text { and } 1(3.0 \%) \text { patient underwent } \\
\text { replacement of the ascending } \\
\text { aorta. CABG coronary artery } \\
\text { bypass grafting; ECMO } \\
\text { extracorporeal membrane } \\
\text { oxygenation; LVAD left } \\
\text { ventricular assist device; MCS } \\
\text { mechanical circulatory support. }\end{array}$ \\
\hline
\end{tabular}

\section{Figure legend}

Figure 1: Comparison of 30-day mortality according to temporary mechanical circulatory support (MCS) strategy. ECMOextracorporeal membrane oxygenation.

Figure 2: Comparison of 30-day mortality according to the timing of temporary mechanical circulatory support implantation.

Figure 3: Comparison of 30-day mortality according to the type of surgery. CABG coronary artery bypass grafting; LVADleft ventricular assist device

Figure 4: Comparison of complication composite outcome during the first 30 postoperative days according to: (A) temporary mechanical circulatory support (MCS) strategy, (B) timing of temporary MCS implantation and (C) type of surgery.CABG coronary artery bypass grafting; ECMOextracorporeal membrane oxygenation; LVAD left ventricular assist device. 

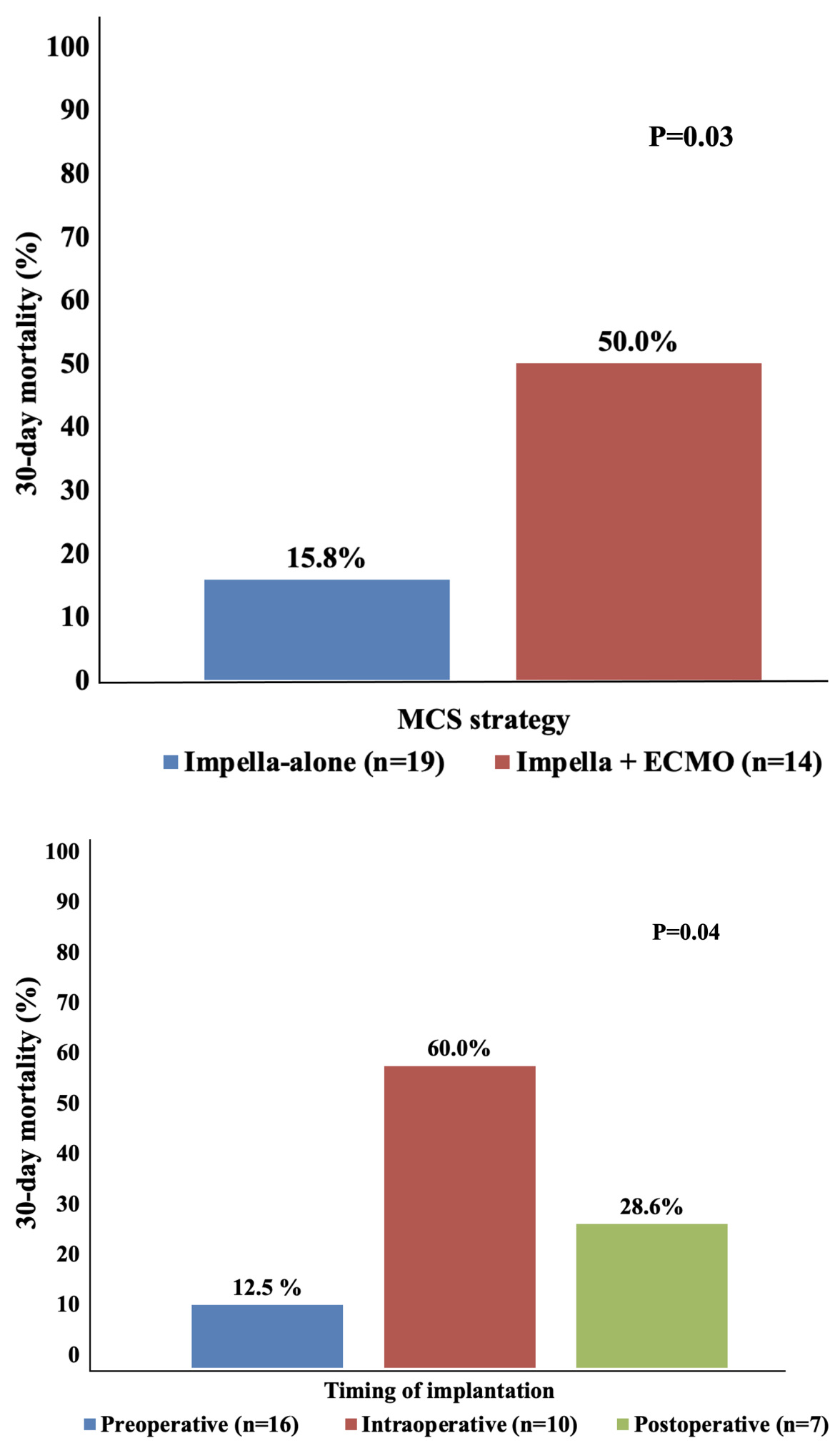

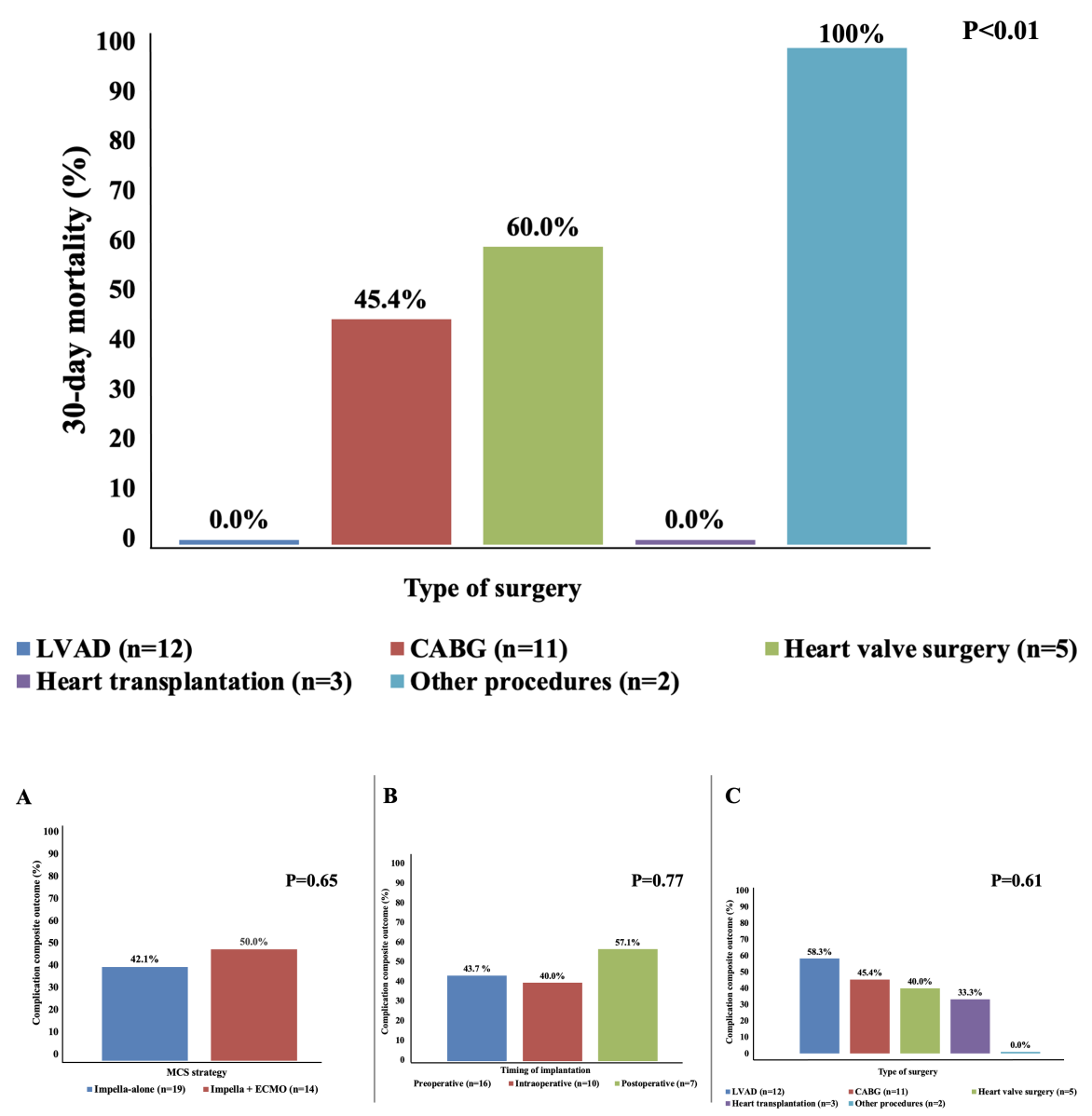